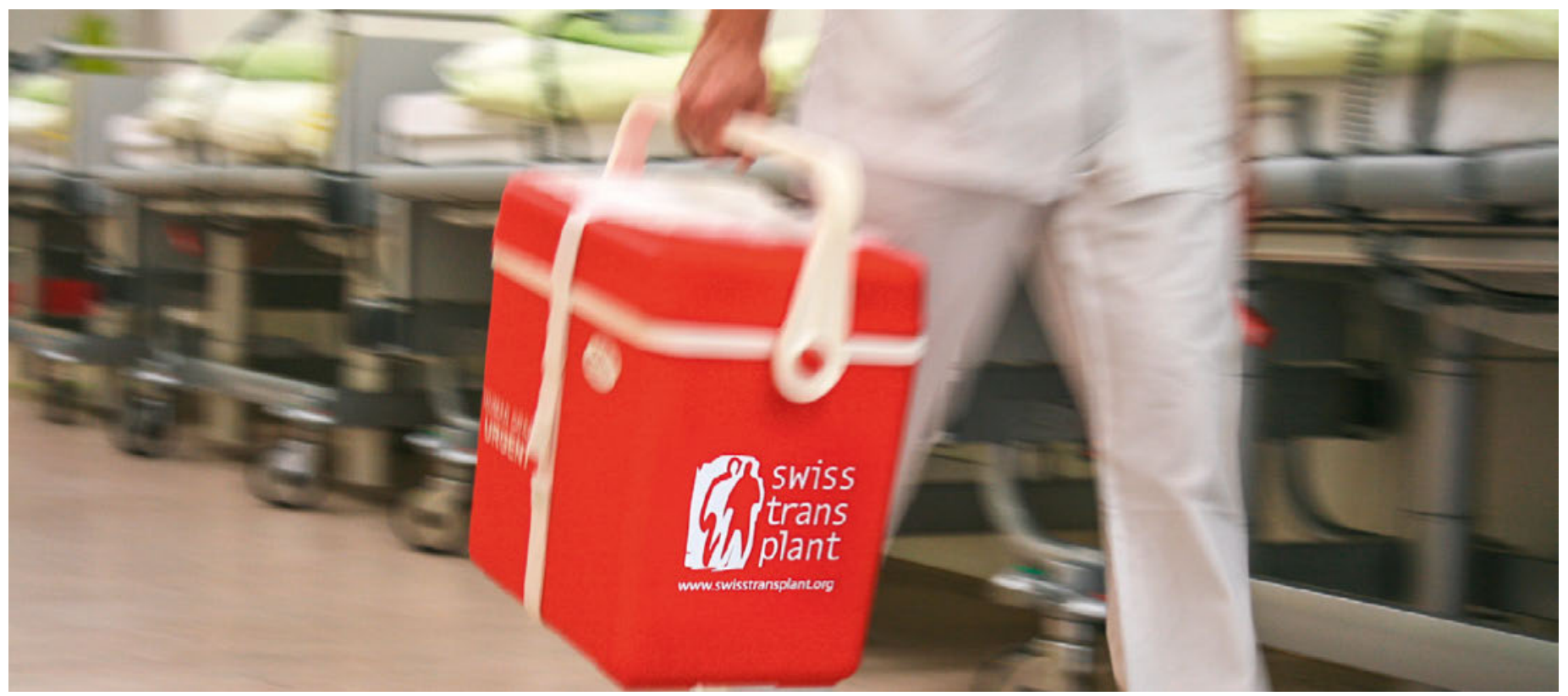

\title{
"Un registre est source de clarté et de sécurité»
}

\section{Matthias Scholer}

Journaliste scientifique

En Suisse, seuls 10\% des donneurs d'organes ont exprimé leur volonté par écrit. Dans tous les autres cas, c'est à la famille qu'il revient de prendre une décision au sujet du prélèvement d'organes. Cela doit à présent changer avec l'initiative populaire "Sauver des vies en favorisant le don d'organes». Si tout se passe comme les auteurs de l'initiative l'espèrent, le consentement présumé, déjà appliqué dans la plupart des pays européens, entrera en vigueur en Suisse.

Fin 2013, la Confédération a lancé le plan d'action «Plus d'organes pour des transplantations». Pouvez-vous en dresser un premier bilan?

Un objectif important du plan d'action était que le don d'organes devienne une thématique établie dans les services d'urgence et de soins intensifs. La collaboration avec les hôpitaux a par conséquent été intensifiée. Grâce à des formations du personnel médical, à l'homogénéisation des processus et à la création de structures organisationnelles claires dans les hôpitaux, les procédures de dons d'organes y sont aujourd'hui appliquées de manière beaucoup plus professionnelle qu'ily a encore quelques années.

Le plan d'action a-t-il aussi eu un effet sur le nombre de personnes prêtes à faire un don?

Malheureusement non. Nous voulons parvenir au même niveau que les pays voisins. Nous visions 20 donneurs d'organes en état de mort encéphalique (donation after brain death, DBD en anglais) par million 
d'habitants. Malheureusement, le niveau reste bas à 13 donneurs par million, malgré différentes mesures complémentaires comme des campagnes d'affichage ou de la publicité télévisée.

\section{Comment expliquez-vous la faible disposition à donner des organes?}

Les causes sont très difficiles à déterminer. Globalement, les Suisses sont plutôt favorables au don d'organes. Cela n'empêche pas $60 \%$ des proches de s'exprimer contre lorsqu'ils doivent prendre une décision à la place de la personne décédée. Ce taux élevé de refus tient sans doute au fait que la famille ne connaît le plus souvent pas les souhaits du défunt et, dans le doute, préfère s'abstenir.

\section{En octobre, la Jeune chambre internationale a lancé} une initiative populaire pour encourager le don d'organes. L'objectif est d'introduire un consentement présumé en Suisse. Comment la collecte de signatures s'est-elle déroulée?

Durant la phase de lancement, l'initiative a été bien accueillie par le public. Une seconde étape avec des journées nationales de collecte est désormais en cours. Les premières expériences montrent qu'il s'agit d'une initiative à laquelle les opposants à un don d'organes de même que les indécis peuvent s'identifier.

Mais n'y a-t-il pas un risque qu'une récolte insuffisante de signatures ou un rejet aux urnes ait un impact négatif durable sur le nombre de donneurs? $\mathrm{Au}$ départ, nous nous sommes également posé cette question. Nous sommes cependant convaincus que des débats à différents niveaux s'amorceront dès la collecte de signatures et permettront de toucher des groupes de population qui ne sont sinon quasiment jamais confrontés au sujet. Le fait qu'un plus grand nombre de personnes réfléchisse au don d'organes et fasse part de son opinion à ses proches peut déjà être vu comme un succès partiel.

\section{En 2015, la Confédération et le Conseil des Etats} se sont exprimés contre un consentement présumé. La Commission nationale d'éthique elle-même est défavorable à cette solution. L'initiative populaire a malgré tout été lancé. S'agit-il d'une réaction de défi? Non, pas du tout. Le consensus politique de l'époque était qu'il fallait attendre les répercussions du plan d'action, qui court jusqu'à la fin 2018. Comme dit, à ce jour, les structures et processus nécessaires ont pu être mis en place au niveau hospitalier. Nous devons à présent discuter de mesures permettant d'augmenter le nombre de donneurs. Pour cela, les modalités du consentement au don d'organes doivent être discutées. Il s'agit au final d'une décision de société, la population doit donc également avoir son mot à dire.

\section{On entend régulièrement que le prélèvement d'or-} ganes chez les personnes en état de mort cérébrale n'est pas acceptable. Ou qu'il a une incidence négative sur le déroulement du décès. Comprenez-vous ces inquiétudes?

Personnellement, je comprends très bien le concept de mort cérébrale. Je suis toutefois conscient que certains collègues remettent en question le concept en tant que tel. C'est précisément pour cela que toute personne qui ne veut pas faire don de ses organes doit pouvoir en faire la mention expresse et sans équivoque dans un registre. La carte de donneur conventionnelle est ainsi modernisée et surtout, l'information est plus facilement accessible.

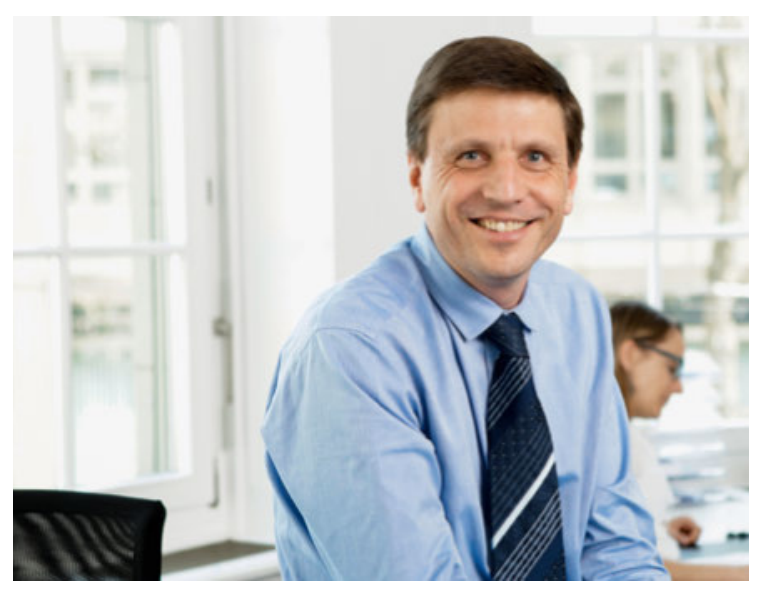

Franz Immer saluerait l'instauration du consentement présumé en Suisse.

La plupart des prélèvements d'organes a-t-elle lieu sur des personnes en état de mort encéphalique?

Nous estimons qu'il se produit dans les services de soins intensifs suisses entre 300 et 350 décès par an répondant aux critères d'un don d'organes en état de mort encéphalique. Depuis 2011, le prélèvement d'organes après un arrêt cardio-circulatoire suite à l'arrêt d'un traitement en unité de soins intensifs a été réintroduit.

\section{Comment le prélèvement d'organes est-il réglementé dans ce cas?}

Il s'agit globalement de patients qui ne répondent pas aux critères de mort encéphalique, mais qui présentent une probabilité de mortalité élevée dans le cadre d'un arrêt de traitement, c'est-à-dire qui seront victimes d'un arrêt cardio-circulatoire dans les 120 mi- 
nutes suivant l'arrêt du traitement. Un prélèvement d'organes peut être effectué chez ces patients après qu'une échocardiographie a prouvé que le cœur ne rejette plus de sang au moins 5 minutes après l'arrêt cardio-circulatoire, puis qu'un constat de décès a été réalisé par deux médecins (principe des quatre yeux) conformément aux directives de l'ASSM.

Si le consentement présumé était instauré, il existerait un registre correspondant. Mais beaucoup de gens ne veulent pas se confronter à la question du don d'organes de leur vivant.

J'ai du mal à concevoir qu'on oblige sa propre famille à s'exprimer sur une question que l'on cherche soimême à éviter.

Un don devrait être un geste altruiste. Dans le cas d'un consentement présumé, ne faudrait-il pas parler d'un don obligatoire de la même manière que l'on parle d'une vaccination obligatoire?

Le changement de système n'entraîne pas d'obligation, simplement un décalage vers une autodétermination active. Chaque personne doit décider pour elle-même si ses organes doivent ou non être mis à la disposition de ses semblables après sa mort. Un registre est source de clarté et de sécurité à cet égard. Cela décharge la famille d'un lourd fardeau. En France, le consentement présumé est en vigueur depuis un certain temps déjà. Un pour cent seulement de la population s'y est expressément opposé à un prélèvement d'organes.
Comme en Italie et en Autriche d'ailleurs, le taux de refus par la famille n'y est que de $30 \%$.

\section{Est-ce que cela signifie que la famille doit prendre la} décision en l'absence d'inscription dans le registre? Oui. Dans un tel cas, une discussion a toujours lieu avec la famille. Elle a toujours pour but d'évaluer le souhait du défunt, car c'est ce qui doit faire foi. Si la famille n'a pas connaissance du fait que le défunt a, de son vivant, exprimé une quelconque forme de refus $\mathrm{du}$ don d'organes, le consentement présumé part du principe qu'il y était favorable.

\section{Une autre approche serait que seuls les donneurs} reçoivent des organes si une greffe s'avérait nécessaire. Que pensez-vous de cette proposition?

Les donneurs doivent être prioritaires. A mon sens, cette façon de faire est justifiée. Elle n'est toutefois pas compatible avec nos bases légales. Le Parlement s'est également exprimé contre cette approche parce qu'elle est discriminante pour la population qui n'a pas réfléchi au sujet du don d'organes. Malheureusement, dans la plupart des cas, on ne se préoccupe de la question que lorsque l'on a soi-même besoin d'un organe. Et à ce moment-là, la plupart des personnes en attente de greffe secouent la tête parce qu'elles ne comprennent pas pourquoi il y a si peu de donneurs.

Crédit graphique

๑) Swisstransplant 